\title{
Nicht-analoge französische und italienische Übersetzungen von restriktiven Partikeln im Roman Die Mittagsfrau von Julia Franck
}

\author{
Martina Nicklaus (Düsseldorf)
}

\begin{abstract}
The present paper discusses the translation of German nur and some synonymous restrictive particles into French and Italian in authorized literary translations. Only non-analogous translations of the restrictive particles have been considered, such as It. si limitò a sorridere for Germ. sie lächelte nur ('she limited herself to smiling' vs. 'she only smiled'). Three types of non-analogous equivalents of nur could be found in the data-base: cleft constructions, verbal periphrases and verb-infinitive combinations. The use of these Italian and French expressions seems to be constantly linked to co-textual features. The description of these nonanalogous equivalents contributes to a better delineation of language-specific preferences concerning the encoding of restriction. Furthermore the description leads to helpful suggestions for translations from French/Italian into German, encouraging translators to substitute e.g. a finite, main verb (It. limitarsi) for a simple particle (Germ. nur) in order to get adequate solutions in German.
\end{abstract}

\section{$1 \quad$ Einleitung}

Die Übertragung von Restriktionsausdrücken wie dt. nur, fr. seulement, ne ... que, it. soltanto, non ... che, stellt Übersetzer mitunter vor Probleme. Zunächst scheinen sich die Ausdrücke als gleichwertige Entsprechungen anzubieten. Sie verfügen alle über dieselben, üblicherweise Partikeln zugeschriebenen Merkmale, d. h. sie sind nicht erfragbar und nicht flektierbar und sie verfügen über relativ hohe syntaktische Mobilität. Sie weisen die gemeinsame restriktive Bedeutung auf, umschreibbar mit Ausschluss von möglichen Alternativen, und sind oft an der Hervorhebung einzelner Satzsegemente beteiligt. Dennoch sind diese Ausdrücke nicht in allen Vorkommen miteinander übersetzbar und gerade solche Fälle sind Gegenstand der folgenden Überlegungen. Dabei sollen, ausgehend von autorisierten literarischen Übersetzungen einerseits und einschlägigen Studien andererseits, konstant beobachtbare Abweichungen von der angenommenen Gleichwertigkeit erfasst werden. Eine solche Abweichung liegt in (1) vor:

(1) Helene schien es, als wollte der Vater sprechen, aber er atmete nur schwer und schluckte [...] (Franck 2007: 43). 
A Helene sembrò che il padre volesse parlare ma respirava $\varnothing$ con difficoltà, deglutiva [...] (Franck/Galli 2008: 39).

wörtlich: [...] aber er atmete $\varnothing$ mit Schwierigkeiten, schluckte [...].

Möglicherweise wollte der italienische Übersetzer Matteo Galli hier zunächst die deutsche Linearisierung, einschließlich des vermeintlich mit $\mathrm{dt}$. nur gleichwertigen it. solo übernehmen: "[...] respirava solo con difficoltà [...]", womit er jedoch den Bezugsbereich der Restriktion verengt hätte, von der Verbalphrase ("atmete ... schwer", eventuell: "atmete ... schwer und schluckte") auf das Adverb schwer, it. con difficoltà. Der Übersetzer verwirft diese Lösung, findet - so die Vermutung - keine alternative Versprachlichung und verzichtet schließlich gänzlich auf den Ausdruck der Restriktion. Auch die französische Übersetzerin des Romans, Elisabeth Landes, vermeidet die sich anbietende Entsprechung (fr. seulement) und entscheidet sich für eine alternative Versprachlichung, für die restriktive verbale Periphrase ne faire que + Inf. (dt., wörtlich, 'nichts tun als'):

Helene crut que son père allait parler, mais il ne fit que soupirer bruyamment, puis il dégloutit [...] (Franck/Landes 2009: 48).

wörtlich: [...] aber er tat nichts als laut atmen, dann schluckte er.

Diese französische Lösung entspricht den Beobachtungen von König (1991: 37), der für Fälle, wo der Bezugsbereich der Restriktionspartikel die gesamte Verbalphrase umfasst, feststellt: "the dummy verb faire has to be inserted in between ne and que."

Die folgenden Ausführungen sind Teil eines Forschungsprojekts $\mathrm{zu}$ literarischer Übersetzung1. Zentrales Anliegen des Projekts ist die Nutzbarmachung von literarischen Übersetzungen für kontrastiv-linguistische Analysen und, umgekehrt, die Nutzbarmachung kontrastiv-linguistischer Befunde für literarische Übersetzung. Es wird angestrebt, Übersetzungsvorschläge aus kontrastiv-linguistischer Sicht anbieten zu können, die stabile Orientierung (keinesfalls präskriptive Anleitung) bei der Übersetzungsarbeit leisten können. Dabei stehen die noch nicht ausreichend in Handbüchern dokumentierten Einzelprobleme, besonders im Rahmen von Linearisierung, im Vordergrund. Über die Tauglichkeit der Vorschläge im Einzelfall wird der Übersetzer ${ }^{2}$ individuell entscheiden, nach Abwägung auch der systematisch nicht vorhersagbaren Aspekte. Besonders für ausdrucksseitige Phänomene, wie etwa die rhythmische Gestaltung des zielsprachlichen Satzes, können angemessene Lösungen nur ad hoc gefunden werden. ${ }^{3}$

$\mathrm{Zu}$ den in Frage kommenden wenig dokumentierten Problemen gehört die Kodierung von Restriktion und besonders ihre einzelsprachlichen Spezifika. Im Folgenden sollen daher, am Beispiel der italienischen und französischen Übersetzungen der deutschen restriktiven Partikeln nur, allein, lediglich, einzig, bloß, erst einige Versprachlichungsspezifika bei Restriktion erfasst und mit sprachwissenschaftlichen Befunden abgeglichen werden. Als Indiz

\footnotetext{
1 Das Projekt Linguistik und literarisches Übersetzen wurde bisher aus Mitteln der Philosophischen Fakultät und des Strategischen Forschungsfonds der Heinrich-Heine-Universität Düsseldorf gefördert.

2 Im folgenden wird - und dies ist politically incorrect, jedoch von der Verfasserin als stilistisch angenehmer betrachtet - grundsätzlich die maskuline Form als generische Form verwendet, obwohl der Anteil der weiblichen Translatoren, auch der weiblichen literarischen Translatoren, den der männlichen bei weitem übersteigt.

3 Vgl. Nicklaus 2013; unter Rhythmus wird hier ein (weitgehend nur vermutbarer) Sprachverarbeitungsrhythmus verstanden.
} 
für solche Versprachlichungsunterschiede werden nicht-analoge Übersetzungen der Restriktionsausdrücke, wie in (1.), gewertet; als "nicht-analog" werden Übersetzungen eingestuft, die mehrteilige, flektierbare und syntaktisch stärker gebundene Restriktionsausdrücke anbieten: Periphrasen, wie in (1), z. T mit eingebettetem seulement/seul/solo ${ }^{4}$, und Verb-Infinitiv-Konstruktionen, wie z. B. fr. se contenter de. Der Einfachheit halber wird die Benennung "Partikel" anstatt "Restriktionsausdruck" nur dann verwendet, wenn von einteiligen, nicht erfragbaren und nicht flektierbaren Restriktionsausdrücken, wie z. B. nur, die Rede ist oder wenn auf die Aussagen und die Terminologie eines Autors Bezug genommen wird. ${ }^{5}$

Der kontrastiven Beispielanalyse werden Beobachtungen zur bisherigen Dokumentation von Restriktion ( $(2)$ sowie eine knappe Beschreibung des Analyse-Instrumentariums vorangestellt: Begriffsbestimmungen zu Äquivalenz, zu Restriktion und Fokussierung ( $\S 3.1$, 3.2, 3.3). Der anschließende Analyseabschnitt (§ 4.) umfasst eine Beschreibung der genutzten bidirektionalen Parallel- und der punktuell eingesetzen Vergleichskorpora (§ 4.1) sowie die Auswertung der Belege (§ 4.2). In der Auswertung werden zunächst die Haupttendenzen in der Übersetzung der deutschen restriktiven Partikeln ( $\S$ 4.2.1) dargestellt, anschließend werden die zwei sich gut abzeichnenden Typen nicht-analoger französischer und italienischer Übersetzungslösungen (§ 4.2.2) hinsichtlich der Übertragung des semantischen (Restriktion) und des informationellen Werts (mögliche Hervorhebung) geprüft. Im Rückgriff auf Satzpaare der entgegengesetzten Übersetzungsrichtung und auf Einzelbeispiele aus Vergleichskorpora werden die Belege der beiden Typen hinsichtlich ihrer Äquivalenz bewertet um abschließend erste und noch vorläufige Übersetzungsempfehlungen formulieren zu können.

\section{Dokumentation von Restriktion}

Nicht immer gelingt die Übertragung von Restriktion bei Übersetzungen. Möglicherweise ist dies auf die zurückhaltende Erforschung der Kategorie und auf die folglich uneinheitliche Dokumentation in den einschlägigen Handbüchern zurückzuführen. Vereinzelt liegen einzelsprachliche Studien zum Ausdruck von Restriktion vor (zum Deutschen besonders Umbach 2001, zum Italienischen Manzotti 1984; Geerts 1977, zum Französischen Raynal 2008, diachronisch: Moignet 1959; im Rahmen von Studien zu Fokuspartikeln z. B.: De Cesare 2010; Andorno 2000; Ricca 1999). Explizit kontrastiv ausgerichtet und somit auch für die Übersetzung von Restriktionsausdrücken interessant ist die Studie von Foolen 1983 (zu den jeweils zentralen deutschen, englischen und niederländischen restriktiven Partikeln). Kontrastive Beobachtungen finden sich weiterhin im Verbund mit Aussagen etwa zu

\footnotetext{
4 Streng genommen handelt es sich bei der Übersetzung einer dt. Restriktionspartikel mit einem fr./it. Spaltsatz und eingeschlossenem seulement/seul/solo immer noch um analoge Übersetzung. Die Einbindung jedoch von seulement/seul/solo in einen neuen, nicht vom Original vorgegebenen syntaktischen Zusammenhang, den Spaltsatz, spricht für die Wertung als zusammenhängende Erscheinung, somit für die Wertung als nicht-analoge Übersetzung.

5 König (1991: 37) spricht implizit auch den angenommenen französischen Äquivalenten seul, ne ... que Partikelstatus zu. Dagegen spricht nicht nur die Zweiteiligkeit von fr. ne ... que (ebenso von it. non ... che), sondern auch die Tatsache, dass fr. seul wie ein Adjektiv angeglichen werden muss, also - partikeluntypisch flektierbar ist.
} 
Fokuspartikeln (König 1991; Sudhoff 2012) oder zu Spaltsätzen (Schöpp 2005; De Cesare/Garassino, im Erscheinen).

In den von Übersetzern gewöhnlich genutzten, formal ausgerichteten Nachschlagewerken fehlen zusammenhängende Darstellungen der Kategorie Restriktion bzw., in anderer Terminologie, der Kategorie Exklusion. Die typischen (jedoch nicht einzigen) sprachlichen Ausdrucksmittel für Restriktion, dt. nur, fr. seul/seulement, it. solo/soltanto, sowie fr. ne ... que und it. non ... che werden auf unterschiedliche Kapitel verteilt ${ }^{6}$. Grammatiken zum Deutschen etwa subsumieren diese restriktiven Ausdrücke meist unter "Partikeln"7, Grammatiken zu den romanischen Sprachen erwähnen sie unter "einschränkende/restriktive Negation"8 und "Adverbien"9. Erst das Bewusstsein für eine übergeordnete Kategorie als tertium comparationis könnte Übersetzern Sicherheit bei der Wahl von mitunter notwendigen, nicht wörtlichen, nicht "analogen" (Doherty 1991: 5) oder nicht "isomorphen" (Nida/de Waard 1986: 32 $)^{10}$ Lösungen geben und ein Umgehen des Restriktionsausdrucks, wie in (1), verhindern.

Nicht umsonst führt der romanistische Sprachwissenschaftler Albrecht (2004: 249, im Rückgriff auf Macheiner 199511) in einem Plädoyer für linguistisches Übersetzertraining, ausgerechnet ein Beispiel zu Restriktion an:

(2) Original: There were only two Americans stopping at the hotel.

Erste Übersetzungslösung: 'Es gab nur zwei Amerikaner, die im Hotel wohnten.'

Zweite, angemessenere Übersetzungslösung: 'Nur zwei Amerikaner wohnten im Hotel.'

Die erste Übersetzung des Beispiels mag naheliegen, wird dem Original jedoch nicht gerecht. Zwar wird die Kombination aus Existenzausdruck mit expletivem Auftakt (There were/Es $g a b)$ und restriktiver Partikel vergleichsweise originalgetreu übernommen, doch das Zusammenspiel dieser beiden Elemente folgt unterschiedlichen Regeln in den beteiligten Sprachen. So scheint, vor dem Hintergrund des Folgetexts, die erste deutsche Version den Rezipienten eher zu verunsichern, während die zweite Version gut interpretierbar ist. Die erste Lösung, so Albrecht, "can be interpreted with a high degree of plausibility in the sense that only two of a greater group of Americans were staying at the hotel" (Albrecht 2004: 249).

6 Auf die terminologische Uneinigkeit schon in Darstellungen zum Deutschen weist auch Eisenberg (2013: 234) hin.

7 Vgl. z. B. Duden ( ${ }^{8} 2009:$ 596), Kap.: "Die Fokuspartikel", Pons (2012: 396), Kapitel: "Die Partikeln".

8 Vgl. z. B. Schwarze ( ${ }^{2}$ 1995: 764), Wilmet 2007: 173 "négation exceptive", dagegen Manzotti 1984: "costrutti esclusivi".

9 Z. B. Grevisse/Goose (2012: §1185), "Adverbes anaphoriques", Lonzi (1991: 349) "avverbi focalizzanti", Kap.: "Il sintagma avverbiale".

10 Der Begriff der Wörtlichkeit/Isomorphie/Analogizität ist problematisch, von den zitierten Autoren werden die Ausdrücke offensichtlich als selbsterklärend eingestuft. Gemeint ist immer eine gewisse Identität zwischen ausgangs- und zielsprachlichem Ausdruck. Außer in der lautlichen Gestalt kann auf allen Sprachebenen annähernde Identität entstehen, auf den Ebenen der Silbenstruktur, der lexikalische Bedeutung, der Linearisierung etc. Kein zielsprachlicher Ausdruck jedoch wird auf allen Ebenen Wörtlichkeit/Isomorphie/Analogizität aufweisen.

11 Die Autorin Judith Macheiner ist identisch mit der hier ebenfalls zitierten Monika Doherty, die ihren Ansatz auch in einer populärwissenschaftlichen Version veröffentlicht hat, unter dem Pseudonym Macheiner (z. B.: Macheiner 1995). 
In der zweiten Lösung richtet sich der Fokus von nur auf das Numerale, dem englischen Original entsprechend: 'Nur zwei der Hotelgäste waren Amerikaner'. Für das Verständnis des Folgetexts, wo von weiteren Hotelgästen die Rede ist, nicht jedoch von einer Gruppe von Amerikanern, ist daher die zweite Lösung passender, während die erste den Leser in eine falsche Interpretationsrichtung führt (vgl. Macheiner 1995: 148-50 und hier $\S$ 4.2.2.1 "Exkurs").

\section{Begriffsbestimmungen}

\section{1 Äquivalenz}

In den neunziger Jahren des letzten Jahrhunderts war Äquivalenz ein Zankapfel, schließlich sogar der "battle-cry" (Neubert 2004: 329) der Translationswissenschaft. Andrew Chesterman, ein Vertreter der empirisch ausgelegten Descriptive Translation Studies, beurteilt das Streben nach Äquivalenz grundsätzlich kritisch, diagnostiziert bereits 1997 den Niedergang ("decline", 1997: 10) des Begriffs; die handlungstheoretisch orientierte Translationswissenschaftlerin Mary Snell-Hornby hält Äquivalenz für eine "Illusion" (Snell Hornby 1986: 13), und der Begründer der Skopos-Theorie Vermeer diagnostiziert bei Äquivalenzbefürwortern abfällig das "Xerox-Syndrom", d. h. das krankhafte Streben nach einer Kopie (Vermeer 1992: 164 N53).

Trotz aller Abgrenzungsschwierigkeiten und Skepsis bleibt der Begriff der Äquivalenz oder Gleichwertigkeit unverzichtbar für jeglichen Sprachvergleich, sei er linguistisch oder translationswissenschaftlich geprägt. Äquivalenz wird hier, dem Projekt-Gedanken folgend, verstanden als Gleichwertigkeit sprachlicher Elemente hinsichtlich eines tertium comparationis in den verglichenen Sprachen.

Das tertium comparationis zu einem sprachlichen Ausdruck setzt sich aus Werten unterschiedlicher Dimensionen zusammen, dazu gehören semantische, stilistische, informationelle, soziokulturelle, textnormbezogene, sprachnormbezogene, kulturbezogene Werte. Idealerweise umfasst das tertium comparationis eines sprachlichen Ausdrucks jene Werte, für die im jeweiligen Translationszusammenhang "Invarianz" (vgl. Albrecht 1990) angestrebt wird. So gehört der stilistische Wert in literarischer Übersetzung, nicht jedoch in Übersetzungen von Gebrauchstexten, zweifelsohne zum Invariantenbündel. Sogar der dezidierteste Gegner jeglichen Abgleichs zwischen Ausgangs- und Zieltext, Gideon Toury, räumt "certain invariance conditions" (Toury 1995: 253) ein. Im Endprodukt "Übersetzung" schließlich wird immer von idealer, kontextfrei ermittelter Äquivalenz abgewichen werden, sowohl durch den systematischen Verzicht auf bestimmte Invarianz-Kriterien im gesamten Text als auch durch kontextuell notwendige Zugeständnisse in Einzelfällen. ${ }^{12}$

Invarianz betrifft im vorliegenden Zusammenhang zunächst den semantischen (Restriktion) und den informationellen Wert (mögliche Fokussierung). Zusätzlich wird punktuell auf den präferenzbezogenen Wert hingewiesen. Dieser Wert wird in Anlehnung an Monika Doherty (z. B. Doherty 1993) ermittelt in Relation zu konstant beobachtbarer Präferiertheit bestimmter

12 Albrecht unterscheidet die "Makroebene" der Übersetzung mit generellen Leitlinien und die zu oft vernachlässigte "Mikroebene" der Übersetzung mit konkreten Angaben zu Einzelproblemen und möglichen Lösungstechniken (vgl. Albrecht ${ }^{2} 2013: 81$ ). 
Versprachlichungen, die vom Leser als "vertraut" wahrgenommen werden. Präferiertheit konzentriert sich in den einzelnen Sprachen infolge stabiler morphosyntaktischer Merkmale und spezifischer Wortschatzstrukturen auf eine Auswahl von jeweils für die Sprachverarbeitung optimalen - präferierten - Kodierungen.

\subsection{Restriktion}

Die semantische Kategorie der Restriktion bzw. der Exklusion (König 1991: 53, 98), drückt Einschränkung bzw. Ausschluss von präsupponierten Alternativen aus (Umbach 2001, König 1991: 53); Restriktion impliziert somit Exhaustivität. Die Alternativen werden grundsätzlich (Andorno 2000: 60; Manzotti 1984; Foolen 1983) oder wenigstens in einem großen Teil der Vorkommen eines Restriktionsausdrucks als graduiert angesehen (Geerts 1977; König 1991: 99s.: "scalar-use"). Von Graduierung der Alternativen wird dann gesprochen, wenn der von nur festgelegte Wert, kontextabhängig, mit dem niedrigsten Grad einer graduierten Reihe assoziiert wird: "I only bought THREE apples." (König 1991: 100). In manchen Vorkommen von Restriktionsausdrücken kann die im Fokus stehende Alternative auch als Bedingung interpretiert werden: "Only a MIRACLE can save us [...]." (König 1991: 101, vgl. hier $\S$ 3.2.2.2).

Unterschieden wird innerhalb von graduierender Restriktion weiterhin zwischen dem quantifizierenden ${ }^{13}$ und dem wertenden Typ (Sudhoff 2012: 207; Foolen 1983: 188; Manzotti 1983: 52) ${ }^{14}$. Erfasst wird damit der Unterschied zwischen Beispielen wie: "Er hat nur ein Auto" (und nicht: ein Auto und ein Fahrrad) und "Er hat nur einen Cinquecento" (und nicht: einen Ferrari). Während manche Partikeln auf eine der beiden Funktionen festgelegt sind, wie z. B. dt. allein, das nach Foolen (1983: 195) und Sudhoff (2012: 210), nur quantifizierend genutzt werden kann, sind andere Partikeln, wie it. solo (vgl. Geerts 1977: 10) oder dt. nur bifunktional, können quantifizierend oder wertend genutzt werden. Die Unterscheidung fällt im Einzelfall mitunter schwer. In (1) etwa ("Helene schien es, als wollte der Vater sprechen, aber er atmete nur schwer [...]") könnte das schwere Atmen als am wenigsten geeignetes Signal für eine kommunikative Absicht bewertet werden, somit der wertende Typ vorliegen. Möglich wäre aber auch, die Sprechabsicht und das hörbare Atmen als verknüpft anzusehen, dann würde durch nur quantifiziert, d. h. es würde eine Komponente eines Ganzen, die Sprechabsicht, ausgeschlossen werden.

Das Alternativen-Paradigma selbst umfasst Ausdrücke von unterschiedlichem Status (De Cesare 2008: 340), z. B. Verbalphrasen (s.o. "wollte ... sprechen", "atmete ... schwer"). Die typischsten sprachlichen Mittel zur Restriktion sind die restriktiven Partikeln. Sie sind syntaktisch am flexibelsten einsetzbar: "They may occur in several positions in a sentence and, as it were, move right through it" (König 1991: 10) ${ }^{15}$ und sind auch semantisch, d. h. für

13 Manzotti (1984: 52) differenziert noch zwischen numerischem ("una non tre") und numerisch-quantitativem Gebrauch ("una macchina, non una macchina e una moto").

14 Foolen und Sudhoff sprechen beim wertenden Typ von "skalierender" Funktion des Restriktionsausdrucks; Manzotti dagegen von qualitativer Funktion, genauer: von "inferiorità qualitativa" beim durch Restriktion hervogehobenen Element.

15 Vgl. ebenso: De Cesare (2008:341), Geerts (1977: 3). Für fr. seul ergibt sich aus der Tatsache, dass nur das grammatische Subjekt im Fokus stehen kann, eine Bindung an die präverbale, bevorzugt satzinitiale Stellung des Subjekts.

ISSN 1615-3014 
die Art von präsupponierten Alternativen, nur wenigen Einschränkungen unterworfen. Letzteres gilt jedoch nicht für die partiellen Synonyme von dt. nur: dt. erst z. B. hat temporale Bedeutung, d. h. die Alternativen sind auf einer zeitlichen Skala angeordnet; dt. allein hat restriktive, nicht jedoch zusätzlich wertende Bedeutung (vgl. Sudhoff 2012: 210), dies gilt auch für dt. einzig. Weiterhin unterstreichen dt. allein, einzig über ihren lexikalischen Bedeutungsanteil das von Restriktion implizierte Merkmal der Exhaustivität.

\subsection{Fokus/Fokussierung}

Auf die Mehrfachbelegung des Begriffs "Fokus" (De Cesare 2008: 343-44) im Zusammenhang mit restriktiven Partikeln und die definitorische Uneinigkeit zu Fokus (vgl. z. B. Schöpp 2005: 85-87, Andorno 2000: 21-33) ist bereits wiederholt hingewiesen worden. Die Verwirrung soll hier durch eine Unterscheidung zwischen Fokus einer Äußerung, Fokus einer Partikel oder eines anderen Restriktionsausdrucks und Fokussierung geklärt werden.

Fokussierung wird hier als vom Produzenten mehr oder weniger bewusst eingesetzte und vom Rezipienten wahrnehmbare, sprachlich manifestierte Hervorhebung definiert, als "segnalazione da parte del parlante dell'importanza di una certa sequenza di discorso." (Sornicola 1988: 178). ${ }^{16}$

Fokussierung "unterläuft" (Oesterreicher 1991: 357) somit sowohl die topic-commentStruktur, als auch die Unterscheidung in bekannte und neue Information. Im definierten Sinn und auf geschriebene Sprache bezogen ist Fokussierung durch restriktive Partikeln dann gegeben, wenn der "ausgewählte" Wert durch zusätzliche Mittel besonderes Gewicht erhält, etwa durch explizite Kontrastierung, und folglich die Aufmerksamkeit besonders bindet.

Der Fokus einer Äußerung wird im Allgemeinen auf informationeller Ebene angesiedelt und grob - als Äußerungssegment mit der kommunikativ relevanten Information definiert. Dabei kann kommunikativ relevante Information z. B. als unvorhersehbare, "unpredictable" Information (Primus 1993: 888; Lambrecht 1994: 207) aufgefasst werden, vergleichbar dem Rhema in anderer Terminologie. Zwischen dem Fokus von Äußerungen und der Distribution von restriktiven Ausdrücken wird ein Zusammenhang vermutet. So werden restriktive Ausdrücke, etwas verwirrend, einerseits als fokussensitiv ${ }^{17}$ (Musan 2010: 43; Skopeteas et al. 2006: 237; Umbach 2001: 165; Andorno 2000: 44), andererseits als fokussierend (Dalmas 2013: 68; Schöpp 2005: 96) beschrieben, d. h. einerseits als fokusabhängig und andererseits als fokuserzeugend. ${ }^{18}$ Dabei wird mitunter ausgeblendet, dass es sich nicht um reine Fokusmarker (König 29: "'pure' focus markers") handelt, sondern um Elemente, deren semantische Kontur zwar gut mit der informationellen Funktion des Äußerungsfokus

16 Eine Umschreibung für "focusing" als "a general term for the assignment of prominence" (Taglicht 1984: 1) erfasst nur die kognitive Voraussetzung für sprachlich manifestierte Fokussierung; ähnlich wie die Definitionen für Fokus von Schöpp (2005: 86): "Informationseinheiten, denen der Sprecher eine ganz besondere Bedeutung zumisst", oder von Wehr (2011: 190): " [...] concept qui est d'une importance particulière pour le locuteur".

17 Sudhoff (2012: 204) beschreibt deutsche und niederländische restriktive Partikeln als mit dem Fokus des Satzes "assoziiert".

18 Auch für die deutschen Partikeln mit modalem Wert stellt Moroni (2010: 127) eine deutliche Affinität zur Position unmittelbar vor dem Fokus einer Äußerung fest. Doherty (1991: 32) spricht den Partikeln im Deutschen durchweg die Funktion des Fokusindikators zu. 
harmoniert, die aber dennoch auch außerhalb des Äußerungsfokus vorkommen können (De Cesare 2010: 113; 2008: 349).

Fokus wird im weiteren nur als Fokus einer restriktiven Partikel verstanden und umfasst jeweils den Wert, der aus einem Paradigma möglicher Werte durch die Operation der Restriktion herausgegriffen wird (König 1991: 12).

\section{$4 \quad$ Analyse}

\subsection{Belegbasis}

Das im Forschungsprojekt und im vorliegenden Zusammenhang verwendete Korpus kombiniert eine parallele mit einer bidirektionalen und einer vergleichenden Perspektive ${ }^{19}$ und folgt damit den von Hansen, Schirra und Teich (2009) formulierten Empfehlungen für korpusbasierte Übersetzungsforschung.

Auswahlkriterien für die Korpustexte sind das Vorliegen französischer und italienischer autorisierter Übersetzungen sowie die Erstveröffentlichung innerhalb der letzten 25 Jahre. Es werden solche Autoren bevorzugt, die Träger der jeweils maßgeblichen nationalen literarischen Auszeichnungen sind (Preis des deutschen Buchhandels, Prix Goncourt, Premio Strega).

Zentral ist in der vorliegenden Studie eine parallele Perspektive (Übersetzungskorpus). Hierfür wurden die ersten 90 Seiten des Romans Die Mittagsfrau (2007) von Julia Franck und die dazugehörigen autorisierten italienischen und französischen Übertragungen systematisch ausgewertet ${ }^{20}$. Aus allen Übersetzungen der deutschen Restriktionspartikeln wurden die nichtanalogen, wie (3) ausgewählt. Sie konstituieren die hier analysierte Datenbasis.

(3) Da hat Mutter nur gelacht, [...] (Franck 2007: 50).

Maman s'est contentée de rire, [...] (Franck/Landes 2009: 56).

Verlässliche Aussagen zu abweichenden Versprachlichungen von Restriktion jedoch sind erst nach erneuter Prüfung, z. B. in originalsprachlichen Vergleichstexten und weiteren Übersetzungskorpora möglich. Nicht umsonst verlangt Schöpp nach einem Vergleich von Fokus-Konstruktionen in Le Petit Prince und der italienischen Übersetzung "weitere Untersuchungen", um "empirische fundierte Aussagen treffen (...) zu können." (Schöpp 2005: 98). Für das hier genutzte Teilkorpus wurden die französischen und italienischen Übersetzungen von nur und seiner (zunächst häufiger erwarteten) literarischen Variante ${ }^{21}$ allein in Francks Die Mittagsfrau erfasst, dabei wurden immer das betreffende Textsegment, mindestens ein Satz, im Original und die dazugehörigen Übertragungen in einem Set zusammengefasst. Ergänzt wurden dann solche Beispiel-Sets, wo in den Übersetzungen

19 Eine reine vergleichende Perspektive nimmt McLaughlin 2008 ein. Sie untersucht die Distribution von Dislokationen in literarischen, original französischsprachigen einerseits und in französischsprachigen literarischen Übersetzungen andererseits, stellt dabei eine ausgesprochen konservative Haltung der Übersetzer fest, die Dislokationen ausschließlich der gesprochenen Sprache zurechnen.

20 Die Texte für das Korpus wurden gescannt und anschließend mit dem Programm FineReader in WordDateien umgewandelt. Die Belege mit restriktiven Ausdrücken wurden in Tabellen extra erfasst.

21 Sudhoff (2012: 210) ordnet dt. allein einer "gehobenen Stilebene" zu. 
französische und italienische restriktive Partikeln einer - mehr oder weniger synonymen Variante von nur im deutschen Original oder sogar einem nicht-restriktiven Ausdruck entsprechen; durch diese Gegenprüfung wurden, in der Reihenfolge ihrer Häufigkeit, die partiellen nur-Synonyme einzig, lediglich, erst, bloß bestätigt und ins Korpus mit aufgenommen, aber auch zwei weitere, überraschendere Entsprechungen gefunden, die nicht in das hier ausgewählte Teilkorpus aufgenommen wurden, da im Deutschen keine Restriktion vorliegt:

(4) [...] vielleicht war sie auch einfach froh [...] (Franck 2007: 42).

[...] forse era solo felice [...] (Franck/Galli 2008: 38).

Die französische Übersetzerin folgt in diesem Fall der deutschen Vorgabe:

[...] à moins qu'elle ne fût bien aise tout simplement (Franck/Landes 2009: 48).

Durch diese Gegenprüfung kann auch eine weitere Bedeutung von seulement, bei verbalem Fokus, belegt werden, die einem deutschen 'überhaupt' entspricht:

(5) [...] war Helene nicht sicher, ob Martha sie überhaupt gehört hatte, vielleicht war sie ganz in ihrer Welt [...] (Franck 2007: 42).

Helene se demanda si elle l'avait seulement entendue ou si elle n'était pas plutôt dans son monde à elle [...] (Franck/Landes 2009: 48).

Helene non era sicura se Marta avesse sentito, forse era completamente immersa nel suo mondo e nei suoi pensieri, [...] (Franck/Galli 2008: 38).

Die bidirektionale Perspektive wird garantiert durch den punktuellen Rückgriff auf originale französische und italienische Texte der Autoren Michel Houellebecq, Jean Echenoz, Dacia Maraini und deren deutsche Übersetzungen. Ein im Übersetzungskorpus entstandener Verdacht auf zielsprachliche Präferenz kann hier überprüft werden. Die oben zitierte Entsprechung nur - se contenter z. B. lässt sich in dt. Übersetzungen nicht nachweisen, se contenter wird immer analog übersetzt, z. B.:

(6) [...] il préférait ne pas se lancer dans la retouche d'images, [...], et se contenter de livrer des clichés techniquement parfaits, mais neutres (Houellebecq 2010: 41).

Er zog es vor, sich nicht mit dem Retuschieren der Fotos abzugeben, (...) und begnügte sich damit, technisch perfekte, ansonsten aber neutrale Negative abzuliefern (Houellebecq/Wittmann 2011: 39).

Das deutsche Ergebnis klingt in der Tat, dank der wiederholten, für das Deutsche untypischen ${ }^{22}$ Subjektivierung durch "zog es vor" - "begnügte sich" schwerfällig; möglich wäre:

Er verzichtete auf das Retuschieren der Fotos, [...] und lieferte lediglich/nur technisch perfekte, ansonsten aber neutrale Negative ab.

22 Blumenthal (1997: 90) stellt fest: Die "Einordnung eines Geschehens in den Wahrnehmungsraum einer Person erweist sich im Sprachvergleich als eine für das Französische typische, für das Deutsche dagegen eher seltene Erscheinung". 
Der vergleichende Blickwinkel wird schließlich eingenommen über die fallweise Einbeziehung originaler französischer und italienischer Texte, sowie, neben Julia Francks Roman, eines weiteren Ausschnitts aus einem orginalsprachlichen deutschen Text, Neue Leben von Ingo Schulze (einschließlich der Übersetzungen); der Rückgriff auf das Deutsche Referenzkorpus des Instituts für deutsche Sprache ${ }^{23}$ erweitert in einem Fall die vergleichende Kontrolle um nicht literarische Belege. Durch diese dritte Perspektive sind Bewertungen von Übersetzungslösungen der Kontrolle durch originalsprachlichen ${ }^{24}$ Gebrauch unterworfen. Frequenz- und Distributionsunterschiede zwischen übersetzter und originaler Sprache sind ein Hinweis darauf, dass beim Übersetzen unterschiedliche Versprachlichungspräferenzen vernachlässigt wurden. So lässt sich das Verb sich begnügen, das im übersetzten Textausschnitt von Houellebecq grundsätzlich als Äquivalent von se contenter eingesetzt (insgesamt fünf Mal) wird, in den beiden deutschen Texten überhaupt nicht finden.

Die für die folgenden Überlegungen zentrale Belegbasis umfasst 89 Vorkommen von restriktiven Partikeln, darunter ist nur die am häufigsten genutzte Partikel (57 Belege), in 16 Fällen liegen partielle, in Semantik, Distribution und Register leicht abweichende Synonyme vor: allein (6), lediglich (5), einzig (4), bloß (1). Das semantisch eingeschränkte erst ist 13 Mal belegt. In drei Fällen liegt ein komplexer restriktiver Ausdruck vor: nichts (oder: nicht etwas) anderes als.

\subsection{Beleganalyse}

Es wird für die Analyse davon ausgegangen, dass alle Lösungen der literarischen Übersetzer grundsätzlich die Folge einer Entscheidung zwischen mehreren, nicht nur originalgetreuen Möglichkeiten darstellt. Immerhin findet sich in allen literarischen Übersetzungen eine Reihe von Beispielen, wo auf nicht analoge Lösungen zurückgegriffen werden musste, mitunter in Missachtung von Invarianten, die sonst im übersetzten Text berücksichtigt wurden. In (7) etwa wurde, um eine satzeinleitende Stellung von seulement zu vermeiden, die grundsätzlich weder in den französischen Übersetzungsbelegen noch in den originalsprachlichen Belegen vorkommt, eine - ko-textuell unwesentliche - Verschiebung des Bezugsbereichs von selten in Kauf genommen:

(7) Nur selten hörten sie zwischen diesen Worten das Fiepen seines Hundes (Franck 2007: 44).

Et ses paroles n'étaient ponctuées que par de rares gémissements du chien (Franck/Landes 2009: 50).

Im Deutschen Originalsatz bezieht sich selten als Adverb auf hörten sie, in der französischen Übersetzung bezieht sich rares (für dt.: selten) als Adjektiv auf gémissements (für dt.: das Fiepen). Näher am Original wäre:

Et ses paroles n'étaient ponctuées que rarement par des gémissements du chien.

23 Konsultiert wurde das Korpus online, über die Web-Applikation Programms COSMAS.

24 Der Vergleich mit originalsprachlichen, funktional vergleichbaren Texten (hier: mit literarischen, narrativen Texten) ist unerlässlich, um die stilistischen Effekte von bestimmten Versprachlichungen kalkulieren zu können. Es wäre jedoch durchaus zu diskutieren, welcher Stellenwert dem Nachempfinden der ausgangssprachlichen Effekte, also einer weitestgehenden Äquivalenz, in der Beurteilung von Übersetzungsqualität zukommt. 
Vermutlich konkurrieren jedoch in dieser Variante die beiden syntaktisch voneinander unabhängigen Einheiten, das Adverbial rarement und das Präpositionalobjekt par des gémissements $d u$ chien, um den informationellen Status als Rhema; tatsächlich sind die Informationen beider Einheiten nicht vorerwähnt. Im vorangegangenen Ko-Text wird lediglich das Sprechen des Vaters und das Zuhören der Mädchen beschrieben:

Sie hörten ihn im Zimmer nebenan auf und ab gehen und wussten, dass er weder Rat noch Hilfe erhielt. Manchmal sagte er etwas, es klang wie Freude! Und wie Gott! Nur selten hörten sie...

Möglicherweise ist nach fr. que (in ne ... que), in unmarkierter Struktur, nur eine informationelle Leerstelle (Rhema) vorgesehen. Um eine optimale Sprachverarbeitung zu garantieren, sollte diese informationelle Leerstelle einer syntaktischen Leerstelle entsprechen. Tatsächlich finden sich in den französischen Übersetzungsbelegen mit dem Restriktionsausdruck ne ... que ausschließlich Beispiele, die nach que lediglich ein Adverbial oder ein Objekt, jeweils eventuell erweitert, anfügen.

\subsubsection{Haupttendenzen der Übersetzung von Restriktionspartikeln}

Im ersten Analysedurchlauf fällt auf, dass im Italienischen in nur wenigen Fällen von der analogen Übersetzung mit solo abgewichen wird; es finden sich sogar fünf Beispiele mit dieser italienischen restriktiven Partikel, die keine Restriktion im Original ersetzen, wie in (4): "[...] forse era solo felice [...]", oder die mit solo eine weitere, mit anderen Mitteln ausgedrückte restriktive Nuance ("das ist alles"/'tutto qua') im Original zusätzlich verstärken, wie etwa:

(8) Er fürchtete sich, das ist alles (Franck 2007: 53).

Lui ha solo paura, tutto qua (Franck/Galli 2008: 39).

It. solo scheint somit tatsächlich über eine den deutschen restriktiven Partikeln annähernd vergleichbare syntaktische Flexibilität zu verfügen. Auch z. B. Schwarze ( $\left.{ }^{2} 1995: 257\right)$, Lonzi (1991: 360s.) und De Cesare (2010: 100) beschreiben für solo und soltanto nur wenige positionelle Einschränkungen; explizit ausschließen möchte Lonzi von den üblichen AdverbPositionen lediglich die Position nach einem Infinitiv (1991: 360). Auf die, möglicherweise mit der syntaktischen Flexibilität begründbare, höhere Gebräuchlichkeit von solo (gegenüber non ... che) weist Schwarze hin $\left({ }^{2} 1995:\right.$ : 765). Gebunden an die syntaktische Flexibilität ist die Möglichkeit, dass Segmente von unterschiedlichstem Status Fokus werden können. Sogar Verbalphrasen, die grundsätzlich selten Fokus von Restriktionsausdrücken werden, sind möglich, wie in (9) (Fokus: schmeckten ... bitter/avevano ... un sapore amaro):

(9) Das Wort klang wie eine Praline, es sollte etwas Edles bezeichnen, und doch schmeckten Pralinen nur bitter (Franck 2007: 49).

Le mot sonnait bien, comme un chocolat fin, il était censé designer quelque chose d'exceptionnel, mais les chocolats laissaient un goût amer (Franck/Landes 2009: 56).

La parola suonava come fosse una praline, doveva designare qualcosa di delicato eppure le praline avevano solo un sapore amaro (Franck/Galli 2008: 45).

Die italienischen Restriktionsausdrücke solamente und soltanto treten vergleichsweise selten auf (viermal bzw. einmal), der zweiteilige Restriktionsausdruck non ... che (viermal) 
ebenfalls. Die Vorliebe des Übersetzers für solo bewirkt die für die lexikalische Ebene wiederholt nachgewiesene Nivellierung in Übersetzungen ("flattening", Chesterman 1997: 70; Laviosa 2003), gewiss oft begründbar mit Zeitdruck.

In der französischen Übersetzung wird satzeinleitendes seulement durchgängig vermieden, mitunter sogar auf Kosten des Ausdrucks von Restriktion (s. a. oben (7)):

(10) Nur wenn Helene eine Strafe verbüßen sollte, [...] (Franck 2007: 47).

Solo se Helene era in punizione [...] (Franck/Galli 2008: 42).

Lorsque Helene devait purger une punition, [...] (Franck/Landes 2009: 53).

Auffällig ist weiterhin, dass fr. seulement überhaupt nur in drei Fällen gewählt wird: einmal innerhalb einer phrase clivée, zweimal in verneinter Form. In einem weiteren, schon angeführten Beispiel wird seulement in der Bedeutung 'überhaupt' eingesetzt (5). In der Mehrheit der Fälle wird Restriktion mit seul (Nominalphrase als Fokus, mit Kongruenz) und ne ... que (Objekte und adverbiale Ausdrücke als Fokus) erfasst.

Typisch etwa:

(11) Einzig die Haushälterin Marja, von den Herrschaften Mariechen genannt und nur wenige Jahre älter als die Mutter, [...] (Franck 2007: 39).

Seule Marja, la bonne, que les maîtres de maison appelaient Mariechen et qui était de peu son aînée, [...] (Franck/Landes 2009: 44).

Solo la domestica Maria, che i padroni chiamavano Mariechen e che aveva solo qualche anno in pìu della madre, [...] (Franck/Galli 2008: 35).

Und:

(12) Einzig für seine Frau hatte er Augen (Franck 2007: 43).

Il n'avait d'yeux que pour sa femme (Franck/Landes 2009: 48).

Lui aveva occhi solo per sua moglie (Franck/Galli 2008: 39).

Verbalphrasen als Fokus der restriktiven Partikeln sind im deutschen Original selten (6 relativ eindeutige Fälle) und führen fast immer zu nicht analogen Übersetzungen (s.o. (1), (3), (9), (13) und, s.u. (14), (23)).

\subsubsection{Nicht analoger Restriktionsausdruck}

Insgesamt zwei Typen nicht analoger, im Übrigen nur monodirektional nachweisbarer Entsprechungen zu den deutschen restriktiven Partikeln kristallisieren sich heraus und werden hier mit "Periphrasen" und "Verb-Infinitiv-Konstruktionen" umschrieben. Zum ersten Typ zählen das schon erwähnte ne faire que (1) und die italienische Entsprechung non fare che sowie Spaltsatzkonstruktionen wie fr. c'est ... qui und it. essere ... $a+$ Infinitiv, zum Teil mit eingeschlossener Restriktionspartikel. Der zweite Typ umfasst Verb-Infinitiv-Konstruktionen wie das ebenfalls schon erwähnte fr. se contenter de + Infinitiv (3) und it. contentarsi di + Inf. 
Für die zum ersten Entsprechungstyp gehörenden Spaltsätze gilt, dass sie hinsichtlich grammatischer Ökonomie der "most costly marking type" (Dufter 2009: 92) sind 25. Dieses Urteil lässt sich auch auf die ebenfalls zum ersten Entsprechungstyp gehörenden faire/farePeriphrasen und auf die Verb-Infinitv-Konstruktionen (zweiter Entsprechungstyp) ausdehnen, wenn auch abgeschwächt, denn diese beiden Konstruktionen fügen keine Sätze hinzu, wie die Spaltsatzkonstruktion, sondern lediglich ein zusätzliches verbales Element als Kopf der Verbalphrase. In formaler Hinsicht ließen sich somit die beiden Entsprechungstypen von nicht analogen Restriktionsausdrücken als aufspaltende Konstruktionen zusammenfassen.

\subsubsection{Erster Typ: Periphrasen (ne faire que/non fare che und Spaltsätze)}

Diese erste Gruppe umfasst 7 Beispiel-Sets.

\section{Faire/fare-Periphrasen:}

(13) Er musste dringend [...], aber er wollte warten. [...] Es war nach Mitternacht, Peter musste nicht mehr, er wartete nur noch (27-28). ${ }^{26}$

Il était minuit passé, Peter n'avait plus envie, il ne faisait plus qu'attendre (30).

Era già passata la mezzanotte, Peter non aveva più bisogno di andare in bagno, non faceva altro che aspettare (25).

$(14)=(2)$ Helene schien es, als wollte der Vater sprechen, aber er atmete nur schwer und schluckte und schickte schließlich die Mädchen aus dem Zimmer (43).

Helene crut que son père allait parler, mais il ne fit que soupirer bruyamment, puis il déglutit, et congédia finalement les fillettes (48).

A Helene sembrò che il padre volesse parlare ma respirava con difficoltà, deglutiva, e alla fine cacciò le ragazze dalla stanza (39).

(15) Helenes Hände kamen und gingen, sie steigerte Marthas Verlangen, noch ein Seufzen, ein einziges wollte sie hören, ihre Hände flogen jetzt sachte über die Haut, berührten nicht mehr alles, nur noch wenig, das wenigste, ihr Verlangen ließ sie schneller atmen, erst Helene, dann Martha, und schließlich beide, es klang wie das Keuchen beim Wringen, wenn man allein am Waschtisch stand [...] (47-48).

Les mains d'Helene allaient et venaient, exacerbant le désir de Martha, un soupir, elle en voulait encore un, rien qu'un seul, ses mains voltigeaient maintenant doucement sur la peau, ne faisant que l'effleurer, juste un peu, le minimum, le désir accélérait leur souffle, d'abord celui d'Helene, puis de Martha, des deux enfin, on entendait un halètement, comme lorsqu'on essore le linge, seule à la table de toilette [...] (54).

Le mani di Helene andavano e venivano, faceva montare il desiderio di Martha, un altro gemito, ne voleva sentire un altro ancora, le mani ora passavano delicate in volo sulla pelle, non toccavano più tutto, solo un po', appena appena, il desiderio le faceva

25 Dies gilt im Besonderen für deutsche Spaltsätze, bei deren Einsatz, anders als im Französischen, mehrere Kongruenzbezüge respektiert werden müssen (vgl. Adam 2013: 44-45).

26 Die Belege des engeren Korpus sind nur noch mit den Seitenangaben für Original (Franck) und Übersetzungen (Franck/Galli; Franck/Landes) zitiert. 
respirare più veloci prima Helene e poi Martha e alla fine entrambe; sembrava il sospiro affannoso di quando si strizzava la biancheria, di quando si stava da sole al lavabo [...] (43).

Die durch die restriktiven Partikeln nur/einzig ausgewählten Werte sind in diesen Belegen immer Teil eines graduierten Paradigmas oder können so interpretiert werden. Die Indefinitpronomina wenig(e), alles (15) suggerieren quantitative Graduierung. Die jeweiligen Werte werden vor dem durch Restriktion ausgewählten Wert genannt: müssen und warten/nur warten (13), sprechen wollen/nur schwer atmen (14). In (15) werden mehrere Alternativen des graduierten Paradigmas benannt, einer davon nach dem ausgewählten Wert: kommen und gehen/sachte fliegen/nicht mehr alles berühren, nur noch wenig/das wenigste [berühren].

Im Original steht in allen Fällen der Wert im Partikelfokus wenigstens einer weiteren Alternative gegenüber, die im unmittelbaren Ko-Text, meist sogar satzintern, verbalisiert, also nicht nur präsupponiert ist. In allen drei Belegen liegt ein mehr oder weniger scharfer inhaltlicher Kontrast zwischen dieser Alternative oder diesen Alternativen (15) und dem Wert des Restriktionsausdrucks vor. Der Kontrast wird durch die explizite Auswahl des einen Werts, also durch die Restriktion, sowie in (13) und (15) zusätzlich durch formale Mittel verstärkt. Für die Auswahl des einen Werts wird die Restriktionspartikel nur eingesetzt. Zur formalen Verstärkung des Kontrasts dient sowohl die Interpunktion als eine bestimmte rhythmische Gliederung. Die Abtrennung mit Kommas isoliert die Alternative(n) und den ausgewählten Wert, die identische Positionierung von Alternative oder Alternativen und ausgewähltem Wert jeweils innerhalb identisch gegliederter Segmente erzeugt eine rhythmische Gliederung: "Peter musste nicht mehr, er wartete nur noch"; (15): "nicht mehr alles, nur noch wenig, das wenigste". ${ }^{27}$ In allen drei Beispielen wird somit durch die Kombination von Restriktion und inhaltlichem Kontrast (14) oder durch die Kombination von Restriktion, Kontrast und formaler Isolierung (von Alternative(n) und ausgewähltem Wert) die Fokussierung des durch nur ausgewählten Werts bewirkt.

In der Übersetzung wird die Fokussierung im Französischen immer, im Italienischen einmal durch die Wahl der faire/fare-Periphrasen nachempfunden. Durch diese Periphrasen ist die Kombination aus Restriktion (ne ... que/non ... che) und Aufspaltung (faire/fare + Infinitiv) bereits vorgegeben. In der faire/fare-Periphrase wird der lexikalische Gehalt eines Verbs als Infinitiv isoliert (hier: attendrelaspettare, soupirer bruyamment/-, effleurer/-); die grammatischen Informationen liefern jeweils finite Formen der Ersatz-Verben faire/fare. Der mehr oder weniger scharfe inhaltliche Kontrast zwischen möglichem und von der Partikel ausgewähltem Wert (z. B. in 13: fr. avoir envie/attendre) wird somit besonders in den französischen Übersetzungen zusätzlich verstärkt, jedoch auf andere Weise als im Deutschen:

27 Hier scheint sich ein Befund von Blumenthal zu bestätigen, der im Deutschen, dank einer systematisch größeren syntaktischen Freiheit, die Vorliebe zur bewussten formalen Gestaltung des Textflusses (mit Hilfe von auch) diagnostiziert und folgert, dass "[...] l'allemand fasse ressortir plus volontiers que le français les relations d'identité, d'analogie ou bien, pour parler avec les logiciens, d'équivalence entre les segments du message (mots, positions, phrases, paragraphes etc.) et cela grâce à l'adverbe auch." (Blumenthal 1985: 145) 
durch die Abspaltung, somit Isolierung, der lexikalischen Komponente des ausgewählten Werts $^{28}$.

\section{Spaltsätze:}

(16) Peter sah an sich hinunter, auf sein Feiertagshemd, erst jetzt erinnerte er sich, dass er zeitig nach Hause kommen sollte (18).

Peter baissa les yeux, s'examina, vit sa chemise du dimanche, et c'est à ce moment seulement qu'il se rappela qu'il devait rentrer tôt (19).

Peter abbassò lo sguardo, físsò la camicia della festa, e solo ora si ricordò che doveva tornare a casa per tempo (16).

(17) Niemand konnte in der Ansammlung eine Ordnung erkennen, einzig die Mutter selbst ahnte, in welchem Stapel sie einen gewissen Zeitungsausschnitt suchen konnte und unter welchem Kleiderhaufen sie die kostbare Spitze abgelegt hat (39).

Nul ne distinguait un ordre dans cet entassement, seule leur mère avait une idée de la pile où elle devait chercher telle ou telle coupure de journal et du tas de vêtements où elle avait rangé la précieuse dentelle sorabe (44-45).

Nessuno era in grado di riconoscere in quell'ammasso di roba un qualche vago ordine, era solo la madre che intuiva in quale pila mettersi a cercare un determinato ritaglio di giornale e sotto quale mucchio di vestiti scovare quel certo prezioso pizzo sorabo. (36)

(18) Es störte ihn nicht, dass Helene die Scheide seines Säbels befühlte, einzig ihre Anwesenheit störte ihn (43-44).

Cela ne le dérangeait pas qu'Helene tâte la lame de son sabre, c'est sa présence qui le dérangeait (49).

Non lo disturbava tanto il fatto che Helene tastasse la lama della sua sciabola, era solo la sua presenza a disturbarlo (40).

(19) Wenn Helene mit Martha über den Kornmarkt ging, dann sahen ihr nicht nur die jungen Männer nach und pfiffen fröhlich, wünschten einen guten Tag. Auch die alten Männer übten Laute, die wie Ächzen oder Grunzen klangen [...] (19).

Quand Helene allait au Kornmarkt avec Martha, les jeunes gens n'étaient pas les seuls à la suivre des yeux, à siffler gaiement et à souhaiter le bonjour. Les vieillards, eux aussi [...] (58).

Quando Helene passava per il Kornmarkt insieme a Martha, non erano solo i giovanotti a guardarla, fischiandole dietro allegramente e augurandogli il buongiorno. Anche i vecchi $[\ldots](46)$.

Die Alternativen zu den durch die restriktiven Partikeln ausgewählten Werten sind, außer in (16), auch hier jeweils im unmittelbaren Ko-Text verbalisiert. Der Fokus-Wert von (17), (18), (19) ist jeweils Teil eines zweiteiligen Paradigmas: niemand/Mutter (17), befühlen/anwesend sein (18), alte und junge Männer/junge Männer (19).

28 Dieser Mechanismus alleine bewirkt bereits eine Fokussierung ("Kontrast-Fokus"), vgl. z. B. Wehr (2011: 210) für das Französische, Roggia (2009: 100) für das Italienische, Adam (2013: 46) für das Deutsche. 
In den Übersetzungen werden, außer in den fr. Versionen von (17) und (19) und der it. Version von (16) die Restriktionsausdrücke in Kombination mit einer Spaltsatz-Konstruktion eingesetzt. Interessant sind die it. Übersetzungen von dt. einzig. Diese dt. Restriktionspartikel unterstreicht Exhaustivität durch ihren lexikalischen Bedeutungsanteil. Sie wird mit einer Kombination aus Spaltsatz und solo übersetzt, eine Kombination, in der De Cesare/Garassino (im Erscheinen) ebenfalls einen besonderen Akzent auf Exhaustivität beobachten: die vom Spaltsatz implikatierte Exhaustivität wird durch solo verstärkt.

Im Original liegt Fokussierung vor. In (17), (18), (19) ist jeweils weniger eine Graduierung als vielmehr ein deutlicher inhaltlicher Kontrast zwischen Alternative und ausgewähltem Wert zu erkennen, der durch eine Form von Negation innerhalb des Paradigmas ausgedrückt wird: niemand/Mutter (17), störte ihn nicht/störte ihn (18), nicht nur/auch (19). In (16) wird mit "Erst jetzt" kein lexikalisch-semantischer Kontrast, sondern ein scharfer, durch asyndetische Verknüpfung der Teilsätze unterstützter inhaltlicher Bruch eingeleitet: im vorangegangenen Text, über den Absatz hinaus, wird der Ablauf einer Plünderung in einem jüdischen Geschäft beschrieben, der im Fokus des Restriktionsausdrucks stehende Wert "jetzt" verweist jedoch nicht auf den Endpunkt dieses Geschehens, sondern auf die vom beschriebenen Geschehen wegführenden Assoziationen des Protagonisten. In allen vier Beispielen bewirkt die Auswahl eines Werts durch Restriktion in Kombination mit einem scharfen inhaltlichen Kontrast zwischen Alternative und ausgewähltem Wert eine Fokussierung des ausgewählten Werts.

In den Übersetzungen wird die Fokussierung großenteils nachempfunden, ähnlich wie bei den faire/fare-Periphrasen. Sowohl die Spaltsätze als auch die Spaltsätze mit zusätzlichem Restriktionsausdruck lösen die ausgewählten Werte (jetzt, die Mutter, ihre Anwesenheit, die jungen Männer) aus der Gesamtproposition heraus und nehmen ihn mit einem Relativpronomen in (16), (17), (18) oder als implizites Subjekt der Infinitivkonstruktion in (18), (19) wieder auf. Der durch Negation etablierte Kontrast zwischen zwei Werten wird somit in allen vier Beispielen zusätzlich verstärkt, entweder durch Restriktion in Kombination mit Abspaltung in (19), in der fr. Version von (16) und in den it. Versionen von (17), (18), oder nur durch Abspaltung in der fr. Version in (18). Nur Restriktion, wie im Deutschen, liegt in der it. Version von (16) und der fr. Version von (17) vor. In den vier Beispielen wird somit in manchen Übersetzungen mit dem Spaltsatz, also erneut mit einer Art Isolierung, ein im Original nicht vorgegebenes, die Fokussierung unterstützendes Mittel eingefügt.

Die Kombination aus Spaltsatz und Restriktion bedarf einiger zusätzlicher Überlegungen. Eine solche Kombination, wie sie im Übrigen auch, abgeschwächt, in (2) vorliegt: "There were only two Americans [...]"29, ist in den Beispielen des deutschen Originals nicht belegt. Dies verwundert nicht, wirken doch schon reine Spaltsätze im Deutschen schnell "stilted" (Dufter 2009: 3), 'gespreizt'; Grund dafür könnte der garantierte Fokussierungseffekt deutscher Spaltsätze (Dufter 2009: 114; indirekt bestätigt in Adam 2013: 4630) sein. In den

29 Das Beispiel (2) kann als Präsentativ-Spaltsatz, als costruzione scissa presentativa (vgl. Roggia 2009: 49) zu den peripheren Ausprägungen des Spaltsatzes gezählt werden.

30 Adam 2013 beschreibt als zentralen "effet de sens" des deutschen Spaltsatzes den Fokussierungseffekt ("mise en exergue", 46), mit und ohne kontrastiver Nuance. Eine weitere zentrale Funktion, die "continuité thématique" 
romanischen Sprachen ist dieser Effekt weniger ausgeprägt, hier liegen neben Fokussierung noch weitere Spaltsatzfunktionen vor, wie etwa die des scene-setting (typisch für das Französische, vgl. Schöpp 2005: 103). Gegen eine Spaltsatznutzung im Deutschen spricht auch die grammatische Komplexität, die "Kongruenz der Kopula" (Dufter 2009: 107) und Angleichung des Relativpronomens. Auch eine mögliche deiktische Überlastung durch bestimmten Artikel und Relativpronomen, wie etwa im folgenden Beispiel (abgewandelter Beleg (17)): "Es war die Mutter selbst, die [...]" könnte stören.

Macheiner schließlich führt die Irritation bei der Kombination aus Existenzausdruck und Restriktion (Präsentativ-Spaltsatz) wie in (2) auf Präferenzen der informationellen Strukturierung im Deutschen zurück, "wo konvex nicht gefragt ist" (Macheiner 1995: 152). Im Deutschen, so Macheiner, ist die mittige Positionierung des Informationsschwerpunkts durch einen Existenzausdruck mit anschließender Restriktionspartikel zum Satzauftakt gerade nicht erwartet, dafür vielmehr konkaver informationeller Aufbau, mit Informationsschwerpunkten am linken und rechten Satzrand, wie in (7):

(7) Nur selten hörten sie zwischen diesen Worten das Fiepen seines Hundes (Franck 2007: 44; Hervorhebung: M.N.).

Der Einschluss eines restriktiven Signals in einen Spaltsatz kann im Deutschen nicht immer dazu dienen, wie im Italienischen, Exhaustivität "explizit" machen (vgl. De Cesare/Garassino, im Erscheinen). Wenn deutsche Spaltsätze grundsätzlich einen Kontrast-Fokus etablieren und damit den betroffenen Sachverhalt als nicht widerrufbar markieren, dann könnte Exhaustivität als semantisch inferierbar angesetzt werden (vgl. Umbach 2001: 168). Restriktion harmoniert folglich dann am besten mit einem deutschen Spaltsatz, wenn der Restriktionsausdruck selbst ebenfalls Exhaustivität garantiert, wie die Partikeln einzig, allein (vgl. unten, Übersetzungsvorschlag zu (22)). ${ }^{31}$

Die Beispiele des Typs Periphrase bestätigen und erweitern eine Aussage von Monika Doherty (alias Judith Macheiner). Für deutsche Übersetzungen aus dem Englischen beobachtet Doherty die Vermeidung der Kombination aus Spaltsatz und Fokussierungspartikel und folgert: "Auf dem Hintergrund der Informationsstruktur der Zielsprache werden die Fokussierungsmittel der Quellensprache als unangemessen, nicht selten als redundant empfunden." (Doherty 1991: 5). Bei den Übersetzungen aus dem Deutschen in romanische Sprachen erscheinen die deutschen Fokussierungsstrategien (Restriktion und bestimmte formale Gestaltungsmittel zur Hervorhebung eines Kontrasts) tatsächlich als "unangemessen", da zwar die Restriktion nachempfunden werden kann, die formalen Gestaltungen des Originals jedoch, eventuell systembedingt, nicht präferiert werden (vgl. N 27).

wird vom Spaltsatz nur in Kombination mit auch ("Sie war es auch, [...]", 49) erfüllt; Belegbasis ist das Deutsche Referenzkorpus - DeReKo des Instituts für Deutsche Sprache.

31 Gut möglich ist im Deutschen wiederum die Verneinung der Restriktion im Spaltsatz (allein 5 Vorkommen in den ersten 100 der aktuellsten Belege für Es ist nur im Deutschen Referenzkorpus DeReKo, jedoch kein Vorkommen ohne Verneinung und kein Vorkommen ohne nur, aber mit Verneinung): "Es ist nicht nur die Besucherzahl, die beeindruckend ist." (Mannheimer Morgen, 25.06.2013: 5). Eine einfache Verneinung des Spaltsatzes widerspricht der Exhaustivität und verwirrt, eine Verneinung mit nur dagegen erhält Exhaustivität. 
Als Beispiel für eine Übersetzungsempfehlung bietet sich ein Beleg aus Echenoz' Je m'en vais an. In (20) hat der Übersetzer den Fokussierungseffekt der fr. phrase clivée im letzten Satz nicht übertragen. Inhaltlich geht es um die Notwendigkeit einer gepflegten Erscheinung vor einem Kunstgalerie-Kunden ("il").

(20) Il a si peur qu'il ne le voit plus, le tableau, n'est-ce pas. Il ne voit plus que vous, le marchand, vous dans vos habits de marchand. Donc c'est votre apparence à vous qu'il va mettre sur le tableau, comprenez-moi (Echenoz 1999: 40).

Er hat solche Angst, dass er das Bild gar nicht mehr sieht. Er sieht nur noch Sie, den Händler, und was der Händler anhat. Das heißt, er überträgt Ihre äußere Erscheinung auf das Bild, verstehen Sie (Echenoz/Schmidt-Henkel 2000: 30).

Im französischen Original wird eindeutig Fokussierung durch den Kontrast zwischen eigentlichem Bild-Inhalt und dem projizierten Bild-Inhalt, d. h. der äußeren Erscheinung des Händlers, erzeugt. Im Deutschen wäre, zur Fokussierung von "Ihre äußere Erscheinung" ein Spaltsatz möglich, jedoch auch eine Übersetzung mit einer restriktiven Partikel (unter Vermeidung einer Wiederholung von nur im vorangegangenen Satz). Auch die Kombination aus beidem bietet sich an:

Das heißt, es ist Ihre äußere Erscheinung, die er auf der Leinwand sieht, [...].

Das heißt, auf der Leinwand sieht er allein/einzig Ihre äußere Erscheinung, [...].

Das heißt, es ist allein Ihre äußere Erscheinung, die er auf der Leinwand sieht, [...].

\subsubsection{Zweiter Typ: Verb-Infinitiv-Konstruktionen}

Der zweite Typ nicht-analoger Entsprechungen umfasst 9 Beispiel-Sets; auch dieser Typ konnte im Korpus nur monodirektional nachgewiesen werden. Im Französischen und, seltener, im Italienischen werden zum Ausdruck der Restriktion Verben eingesetzt, die restriktiven Charakter haben oder im weitesten Sinne restriktiv deutbar sind. Angeschlossen wird jeweils, in infiniter Form, das im Original im Fokus stehende Verb oder ein Verb, das den semantischen Gehalt anderer Segmente des Originalsatzes aufnimmt. Die verwendeten verbalen Ausdrücke sind: fr. se contenter, se borner/it. limitarsi und fr. devoir, falloir/it. bastare. Im Original liegen als Fokus entweder Verbalphrasen vor (drei Belege: lächelte/hat gelacht/sich sanft und leise in Selbstgesprächen ärgerte), ganze Nebensätze (drei Belege: wenn [...]) oder komplexe adverbiale Präpositionalphrasen (zwei Belege: vom Flattern seiner Augenlider / bei genauem Hinsehen und mit Hilfe einiger Rückschlüsse von der sonstigen Physiognomie).

1. Fr. se contenter, se borner/it. limitarsi

(21) Erstaunt blickte Peter sie an, aber sie lächelte nur und stieß ihre Nadel in das Leinen (10).

Étonné, Peter l'avait regardée, mais elle s'était contentée de sourire et de piquer son aiguille dans le lin (11).

Peter la osservò stupito, ma lei si limitò a sorridere, infilando l'ago nella stoffa (10). 
$(22)=(3)$ Da hat Mutter nur gelacht, so gelacht, dass ich dachte, es stimmt, was Vater vermutet (50).

Maman s'est contentée de rire, mais de rire, et je me suis dit que papa avait touché juste (56).

E nostra madre allora è scoppiata a ridere, a ridere così forte che mi è venuto da pensare, forse quello che sospetta nostro padre non è così sbagliato (45).

(23) Ihr fiel jetzt ein, dass der Vater sie zwar neuerdings die Buchhaltung für die Druckerei machen ließ, sich aber lediglich sanft und leise in Selbstgesprächen darüber ärgerte, wenn Helene irgendwo einen Fehler gefunden hatte (55). [Helene erwartet ein laut ausgesprochenes Lob. M.N.]

Elle se rappelait alors que s'il lui confiait depuis peu la comptabilité de l'imprimerie, son père se bornait à monologuer irrité à voix basse, quand elle découvrait une erreur quelque part (63).

Solo allora le tornava in mente che, sebbene il padre negli ultimi tempi le avesse affidato la contabilità della tipografia, finiva per arrabbiarsi tutte le volte che Helene scovava un qualche errore, lasciandosi andare a monologhi a mezza voce. (50). [Missverständnis des it. Übersetzers, der Fokus des Restriktionsausdrucks ist vertauscht. M.N.]

In allen drei Belegen sind die Alternativen zum ausgewählten Wert lediglich präsupponiert, also nicht im Ko-Text genannt. Der Fokus des dt. Restriktionsausdrucks ist eine Verbalphrase. Das Französische verlangt in diesen Fällen laut König (vgl. oben, 1991: 37) die Periphrase ne faire que. Grevisse ist hier toleranter und sieht die Notwendigkeit für ne faire que nur "lorsque la restriction porte sur un verbe à un temps simple" (Grevisse 2012: § 1018), also nur bei nicht zusammengesetzten Tempusformen. Die Übersetzerlösungen verweisen auf eine weitere Möglichkeit zur Restriktion von Verbalphrasen, auf die Verwendung von restriktiven, reflexiven Verben wie se contenter, se borner und contentarsi, limitarsi. Die einsprachigen Wörterbücher unterstützen diese Lösung und erfassen die betreffenden Verben auch in einer lexikalisch blassen, restriktiven Bedeutung ('seulement'/'soltanto', vgl. Petit Robert 2008; Zingarelli 201032, s. v.). Die Beispiele erinnern erneut, nicht zuletzt durch die Reflexivität der Verben, an eine Tendenz zur "Subjektivität", die für das Französische u. a. von Blumenthal im Zusammenhang mit Perspektivierung von Sachverhaltsdarstellungen (1997: 90; vgl. N 22) belegt worden ist. In den obigen Beispielen ist es die Darstellung des verbalen Sachverhalts als nicht nur menschliche Handlung, sondern vor allem als bewusste menschliche Handlung, die eine Subjektivierung bewirkt. ${ }^{33}$

In den Belegen liegt keine Fokussierung (im hier definierten Sinn) des durch die Restriktion ausgewählten Werts vor.

32 Konsultiert wurden die jeweils aktuellsten digitalisierten Versionen der beiden Wörterbücher. Es handelt sich nicht um frei zugängliche Web-Versionen, sondern um die digitalisierten und erweiterten Ausgaben der PrintVersionen.

33 Interessanterweise würde in den ersten beiden Beispielen die Übersetzung mit seulement/soltanto eine ironisch-abwertende Nuancierung bewirken: souriait seulement/ sorrideva soltanto. Die Bevorzugung der Verbalkonstruktionen ist hier also auch semantisch motiviert. 


\section{Fr. devoir, falloir/it. bastare}

(24) Peter blinzelte, er hoffte, die Möwe werde allein vom Flattern seiner Augenlider aufgescheucht und flöge davon (9).

Peter clignait des yeux, il espérait que le seul battement de ses paupières l'effaroucherait, la ferait s'envoler (9).

Peter strizzò gli occhi sperando che lo sbattere delle palpebre bastasse a impaurire il gabbiano, e a farlo volare via (9).

(25) Allein, wenn er sich das Funkeln ihrer Augen vorstellte, deren Farbe zwischen Grün und Braun und Gelb wechselte, lief ihm ein Schauer und des Verlangens und Wohlseins in der Welt über den Rücken (75).

Rien qu'en se remémorant l'éclat de ses yeux dont la couleur variait, passant du vert au brun ou au jaune, un frisson de désir et de bien-être terrestre lui parcourait le dos (85).

Gli bastava solo immaginarsi il luccicchio dei suoi occhi, il cui colore cangiava fra il verde, il castano e il giallo, e subito la schiena era percorsa da un brivido di desiderio e di benessere a questo mondo (66).

(26) Erst wenn einer sich traute und sie fragte, ob er sie einmal zum Kaffee einladen dürfte, und sie lachend ablehnte, sie gehe niemals mit Kunden einen Kaffee trinken, rückte die Entscheidung für einen kleinen Druckauftrag näher (52).

Il fallait que l'un d'eux se decide à lui demander s'il pourrait l'inviter à boire un café et qu'elle refuse en riant, alléguant qu'elle n'allait jamais boire de café avec les clients, pour que se dessine la perspective d'une petite commande d'impression (59).

Solo quando uno di loro si faceva coraggio e le domandava se poteva magari una volta invitarla a prendere un caffè e lei con una risata rifiutava dicendo che non andava mai a prendere un caffè con i clienti, solo allora si arrivava a commissionarle l'incarico di un lavoretto a stampa (47).

(27) Erst wenn der Stein geraume Zeit neben ihm auf dem Laken gelegen hatte, konnte er die Augenhöhle wieder kühlen (75).

La pierre dut reposer un bon moment à côté de lui sur le drap avant de pouvoir refraîchir de nouveau son orbite (82).

Solo dopo che era stata un po' di tempo sul lenzuolo accanto a lui, la pietra poteva tornare a raffreddare la cavità oculare (64).

La pierre dut reposer un bon moment à côté de lui sur le drap avant de pouvoir refraîchir de nouveau son orbite (82).

(28) Ja, das Gesicht sei wunderbar verheilt, man könne wohl nur bei genauem Hinsehen und mit Hilfe einiger Rückschlüsse von der sonstigen Physiognomie erkennen, wo sich einmal dieses rechte Auge befunden habe (75).

Oui, son visage avait merveilleusement cicatrisé, et il fallait l'examiner soigneusement pour pouvoir déduire du reste de sa physionomie l'emplacement peu reconnaissable où se trouvait jadis cet oeil droit (84). 
Sì, il volto era meravigliosamente guarito e solo guardando meglio e con l'aiuto di alcune congetture basate sul resto dei suoi connotati era possibile riconoscere il punto esatto dove un tempo si trovava l'occhio destro (66).

In allen Beispielen werden keine Alternativen zu dem im Partikel-Fokus stehenden Wert im Ko-Text verbalisiert. Mit dem Verb bastare ('ausreichen') als Ausdruck von Restriktion liegt eine spezifisch italienische Option vor, die auch in der italienischen Übersetzung eines Texts aus dem Vergleichskorpus viermal bestätigt wird, z. B.:

(29) Wenn es jetzt bloß nicht aufhört, dachte ich, wenn es nur weitergeht (Schulze 2005: 26).

Purché non finisca ora, ho pensato, basta che si vada avanti (Schulze/Cambi 2007: 27).

Auffällig ist, dass in (24) bis (28) der im Fokus von nur und von allein stehende Ausdruck immer eine Bedingung erfasst. In Anlehnung an eine von König vorgenommene Unterscheidung (1991: 102) handelt es sich um notwendige Bedingungen in den Belegen mit der Partikel allein (24), (25), auch in dem eben zitierten Beleg (29), und um hinreichende Bedingungen in den restlichen Belegen dieser Gruppe. Für die italienischen Übersetzer deckt bastare den ersten Bedingungstyp offenbar besser ab als solo, eine aufgrund der Bedeutung von bastare nachvollziehbare Lösung. Der zweite Bedingungstyp wird wieder mit der Partikel solo wiedergegeben. Das Französische dagegen versprachlicht den ersten Bedingungstyp mit den Restriktionsausdrücken seul (hier: attributiv) und rien que ${ }^{34}$, dafür den zweiten Bedingungstyp nicht-analog, mit den Modalverben devoir oder falloir ('müssen').

In den Belegen liegt keine Fokussierung (im hier definierten Sinn) des durch die Restriktion ausgewählten Werts vor.

Als Beispiel für eine Übersetzungsempfehlung bietet sich der folgende Beleg aus Marainis Marianna Ucría an.

(30) Non ha portato la candela con sé; il naso basta a guidarla fra corridoi, scale, strettoie, cunicoli, ripostigli, bugigattoli, rampe improvvise e scalini traditori (Maraini 1990: 74).

Sie hat keine Kerze mitgebracht; doch ihre Nase reicht aus, um sie durch Korridore, über Treppen, durch Engpässe, Stollen, Abseiten, Schlupfwinkel, über plötzliche Rampen und trügerische Stufen zu führen (Maraini/Kienlechner 1993: 74).

Die vorgeschlagene deutsche Übersetzung klingt umständlich. Wenn die oben sich angedeutete Äquivalenz allein - bastare angesetzt wird, wäre möglich:

Sie hat keine Kerze mitgebracht; allein ihre Nase führt sie durch Korridore, über [...].

Diese Formulierung würde auch der Linearisierung im italienischen Original folgen und die Endstellung des Infinitums ("[...] trügerische Stufen zu führen.") umgehen.

34 Laut Moignet (1959: 170) ist rien que im Français moderne zum "équivalent appuyé" von ne ... que geworden; auch im Petit Robert (2008-, s. v.) wird als Bedeutung seulement angegeben. 


\section{Schluss}

Vorläufig lassen sich die Beobachtungen wie folgt in Thesen zusammenfassen.

Wird die im Fokus der restriktiven Partikel stehende Konstituente durch satzinterne oder absatzinterne Kontrastierung von Alternative(n) und ausgewähltem Wert, sowie durch zusätzliche formale Mittel (rhythmische Gliederung, Gliederung durch Interpunktion) fokussiert, ist ein Spaltsatz oder die Kombination aus Spaltsatz und restriktiver Partikel eine passende französische und italienische Entsprechung.

Drückt das im Fokus der restriktiven Partikel stehende Segment eine notwendige (typische Partikel: dt. allein) oder hinreichende Bedingung (typische Partikel: dt. erst (wenn)) aus, werden zum Teil Verb-Entsprechungen (anstatt Partikeln) angesetzt, d. h. es wird im Italienischen der erste Bedingungstyp mit it. bastare erfasst, im Französischen wird der zweite Typ mit fr. devoir oder falloir erfasst.

Drückt das im Fokus der restriktiven Partikel stehende Segment dagegen eine Handlung aus, bei agentivischem Subjekt, kann die romanische Entsprechung ein reflexiver Verbalausdruck wie fr. se contenter/se borner, it. contentarsi/limitarsi sein.

Um diese drei an den nicht analogen Übersetzungslösungen abgelesenen Tendenzen als konstant beobachtbare Versprachlichungspräferenzen $\mathrm{zu}$ bestätigen, müssen erweiterte empirische Studien, dann auch unter systematischer Einbeziehung der bidirektionalen und der vergleichenden Perspektive durchgeführt werden.

Entschieden werden müsste ebenfalls, wie sich diese Präferenzen an konstante Vorgaben der einzelnen Sprachen zurückbinden lassen. So könnten morphosyntaktische System-Vorgaben ("Parametrisierungen", Doherty 1993), und genauer: die vergleichsweise rigiden syntaktischen Einschränkungen im Französischen, für die Vermeidung der satzeinleitenden Position von seulement verantwortlich sein. Auch Prinzipien der Linearisierung könnten eine Rolle spielen: so könnte der häufige Gebrauch von it. solo mit einem Bedürfnis nach deutlicher informationeller Markierung zusammenhängen und somit auf die bereits namhaft gemachte und zum Teil belegte hohe Gewichtung der Informationsstruktur bei der Linearisierung im Italienischen (Lambrecht 1994: 24; Ulrych 1997: 143; Nicklaus 2013; Ravetto 2011: 214; Blühdorn 2012: 328) zurückgeführt werden.

\section{Literatur}

\section{Primärquellen}

Echenoz, Jean (1999): Je m'en vais, Paris: Minuit.

Echenoz, Jean/Schmidt-Henkel, Hinrich (2000): Ich gehe jetzt. Berlin: Berlin Verlag.

Franck, Julia (2007): Die Mittagsfrau. Frankfurt a. M.: Fischer.

Franck, Julia/Galli, Matteo (2008): La strega di mezzogiorno. Firenze: Le Lettere.

Franck, Julia/Landes, Élisabeth (2009): La femme de midi. Paris: Flammarion.

Houellebecq, Michel (2010): La carte et le territoire. Paris: Flammarion.

Houellebecq, Michel/Wittman Uli (2011): Karte und Gebiet, Köln: Dumont.

Maraini, Dacia (1994 [ $\left.\left.{ }^{1} 1990\right]\right):$ La lunga vita di Marianna Ucría. Milano.

Maraini, Dacia/Kienlechner, S. (1993): Die stumme Herzogin. München: Piper. 
Schulze, Ingo (2005): Neue Leben, Berlin: Berlin Verlag.

Schulze, Ingo/Cambi Fabrizio (2007): Vite nuove, Milano: Feltrinelli.

\section{Sekundärquellen}

Adam, Séverine (Hrsg.) (2013): 'Informationsstrukturen' im gesteuerten Spracherwerb: Französisch - Deutsch kontrastiv. Frankfurt a. M., PL Acad. Research.

Adam, Séverine (2013): "Ressemblance formelle, différence fonctionnelle - l'exemple des clivées". In: Adam, Séverine (Hrsg.): 'Informationsstrukturen' im gesteuerten Spracherwerb: Französisch - Deutsch kontrastiv. Frankfurt a. M., PL Acad. Research: 3366.

Albrecht, Jörn (1990): "Invarianz, Äquivalenz, Adäquatheit". In: Reiner, Arntz/Gisela, Thome (Hrsg.): Übersetzungswissenschaft. Ergebnisse und Perspektiven. Festschrift für Wolfram Wilss zum 65. Geburtstag. Amsterdam/Philadelphia, Benjamins: 71-81.

Albrecht, Jörn (2004): "The different branches of descriptive linguistics and translation". In: Kittel, Harald et al. (Hrsg.): Übersetzung, Translation, Traduction. Vol 1. Berlin, de Gruyter: 243-259.

Albrecht, Jörn (2013): Übersetzung und Linguistik. Tübingen, Narr.

Andorno, Cecilia (2000): Focalizzatori fra connessione e messa a fuoco. Il punto di vista delle varietà di apprendimento. Milano, FrancoAngeli.

Blühdorn, Hadarik (2012): "Zur Syntax adverbialer Satzverknüpfungen: Deutsch - Italienisch - Portugiesisch". In: Gunkel, Lutz (Hrsg.): Deutsch im Sprachvergleich. Grammatische Kontraste und Konvergenzen. Berlin/New York, de Gruyter Mouton: 301-331.

Blumenthal, Peter (1985): "AUSSI et AUCH: deux faux amis?". Französisch heute 2: 144-150.

Blumenthal, Peter (1997): "Satzmuster im Deutschen und Italienischen". In: Welte, Werner (Hrsg.): Sprachtheorie und angewandte Linguistik. Festschrift für Alfred Wollmann zum 60. Geburtstag. Tübingen, Narr: 147-159.

Chesterman, Andrew (1997): Memes of Translation. Amsterdam/Philadelphia: Benjamins.

Dalmas, Martine (2013): "Strategien der Fokussierung: eine 'spannende' Geschichte - und eine Revidierung mancher Vorurteile". In: Adam, Séverine (Hrsg.): 'Informationsstrukturen' im gesteuerten Spracherwerb: Französisch - Deutsch kontrastiv. Frankfurt a. M., PL Acad. Research: 67-77.

De Cesare, Anna-Maria (2008): "Gli avverbi paradigmatizzanti". In: Ferrari, Angela et al. (Hrsg): L'interfaccia lingua-testo. Natura e funzioni dell'articolazione informativa dell'enunciato. Alessandria, Dell'Orso: 340-361.

De Cesare, Anna-Maria (2010): "On the focusing function of Focusing Adverbs: A discussion based on Italian data". Linguistik online 44/4: 100-116.

De Cesare, Anna-Maria/Garassino, Davide (im Erscheinen): "On the status of exhaustiveness in cleft sentences: A data-driven and cross-linguistic study of English also-/only-clefts and Italian anche-/solo-clefts. Folia Linguistica.

Doherty, Monika (1991): "Spaltsatz oder Fokussierungspartikel". In: Kohrt, Manfred/Küper, Christoph (Hrsg.): Probleme der Übersetzungswissenschaft: 5-37.

Doherty, Monika (1993): "Parametrisierte Perspektive". Zeitschrift für Sprachwissenschaft 12/1: 3-38. 
Duden ( $\left.{ }^{8} 2009\right):$ Duden. Die Grammatik. Unentbehrlich für richtiges Deutsch. Mannheim, Duden-Verlag.

Dufter, Andreas (2009): "Clefting and discourse organization. Comparing Germanic and Romance". In: Dufter, Andreas/Jacob, Daniel (Hrsg.): Focus and Background in Romance Languages. Amsterdam/Philadelphia, Benjamins: 83-121

Eisenberg, Peter ( $\left.{ }^{4} 2013\right)$ : Grundriss der deutschen Grammatik. Vol 2: Der Satz. Stuttgart/Weimar, Metzler.

Foolen, Ad (1983): Zur Semantik und Pragmatik der restriktiven Gradpartikeln: only, nur und maar/alleen. In: Weydt, Harald (Hrsg.): Partikeln und Interaktion. Berlin, Schmid: 188-202.

Geerts, Walter (1977): "On the semantics of Italian adverbial solo". Archivum linguisticum 8/1: 3-12.

Grevisse, Maurice/Goosse, André (2012): Le bon usage: grammaire française. Bruxelles, De Boeck-Duculot.

Hansen-Schirra, Silvia/Teich, Elke (2009): "55. Corpora in human translation". In: Lüdeling, Anke (Hrsg.): Corpus Linguistics. Vol. 2. Berlin/New York, de Gruyter Mouton: 1159-1175.

König, Ekkehard (1991): The Meaning of Focus Particles: A Comparative Perspective, London/New York, Routledge.

Lambrecht, Knud (1994): Information Structure and Sentence Form. Topic, Focus and the Mental Representations of Discourse Referents. Cambridge, Cambridge University Press.

Laviosa, Sara (2003): "Corpus and simplification in translation". In: Petrilli, Susan (Hrsg..): Translation, Translation. Amsterdam/New York, Rodopi: 153-162.

Lonzi, Lidia (1991): "Il sintagma avverbiale". In: Renzi, Lorenzo/Salvi, Giampaolo (Hrsg.): Grande grammatica italiana di consultazione. Vol. 2: I sintagmi verbale, aggettivale, avverbiale. La subordinazione. Bologna, il Mulino: 341-412.

Macheiner, Judith (1995): Übersetzen. Ein Vademecum. Frankfurt a. M., Eichborn-Verlag.

Manzotti, Emilio (1984): "Costrutti esclusivi e restrittivi in italiano". Vox Romanica 43: 50-80.

McLaughlin, Mairi (2008): "(In)visibility: dislocation in French and the voice of the translator". In: French Studies 62/1: 53-64.

Moignet, Gérard (1973) [ $\left.{ }^{1} 1959\right]$ : Les signes de l'exception dans l'histoire du français. Genève, Droz

Moroni, Manuela Caterina (2010): Modalpartikeln zwischen Syntax, Prosodie und Informationsstruktur. Frankfurt a. M., Lang.

Musan, Renate (2010): Informationsstruktur. Heidelberg, Winter.

Neubert, Albrecht (2004): "Equivalence in translation". In: Kittel, Harald et al. (Hrsg.): Übersetzung, Translation, Traduction. Vol 1. Berlin/New York, de Gruyter Mouton: 330-340.

Nicklaus, Martina (2013): "La pazienza tedesca, l'impazienza italiana: un confronto di preferenze die serializzazione in italiano e tedesco. Deutsche Geduld, italienische Ungeduld: Ein Vergleich Serialisierungspräferenzen im Italienischen und Deutschen". In: Schafroth, Elmar et al. (Hrsg.): Italien, Deutschland, Europa: Kulturelle Identitäten und Interdependenzen/Italia, Germania, Europa: fisionomie e interdipendenze. Oberhausen, Athena: 252-273. 
Nida, Eugene/de Waard, Jan (1986): From One Language to Another. Functional Equivalence in Bible Translation. Nashville, Nelson.

Oesterreicher, Wulf (1991): "Verbvalenz und Informationsstruktur". In: Koch, Peter/Krefeld, Thomas (Hrsg.): Connexiones Romanicae. Dependenz und Valenz in romanischen Sprachen. Berlin, de Gruyter: 349-384.

Petit Robert (2008): Le Petit Robert de la langue française: dictionnaire alphabétique et analogique de la langue française. Paris, Dictionnaires Le Robert. [Online-Version, Letzter Zugriff: 15.08.2014].

Pons (2012): Die deutsche Grammatik. Das umfassende Nachschlagewerk. Augsburg, Weltbild.

Primus, Beatrice (1993): "Word order and information structure: a performance-based account of topic positions and focus positions". In: Jacobs, Joachim/von Stechow, Arnim (Hrsg.): Syntax. Ein internationales Handbuch zeitgenössischer Forschung. Vol. 1. Berlin, de Gruyter: 880-896.

Ravetto, Miriam (2011): "Die deutschen Kausalkonnektoren und ihre italienischen Äquivalente: Syntax und Infomationsstruktur". In: Lavric, Eva/Pöckl, Wolfgang/Schallhart, Florian (Hrsg.): Comparatio delectat. Akten der VI. Internationalen Arbeitstagung zum romanisch-deutschen und innerromanischen Sprachvergleich (Innsbruck 3.-5. 2008). Frankfurt a. M., Lang: 201-215.

Raynal, Céline (2008): La restriction en français: trois études sémantiques. Paris, Université Paris 7 - Denis Didérot. Online abrufbar:http://tel.archives-ouvertes.fr/docs/00/34/51/85/ PDF/CRaynal_these.pdf. [Thèse de doctorat]

Ricca, Davide (1999): "Osservazioni preliminari su focalizzatori in italiano". In: Dittmar, Norbert/Giacalone Ramat, Anna (Hrsg): Grammatik und Diskurs. Studien zum Erwerb des deutschen und Italienischen. Tübingen, Stauffenburg: 145-163.

Roggia, Carlo Enrico (2009): Le frasi scisse in italiano. Struttura informativa e funzioni discorsive. Genève, Slatkine.

Schöpp, Frank (2005): "Fokus-Konstruktionen im Italienischen - mit Vergleichen zum Französischen". Beiträge zur Fremdsprachenvermittlung 43: 85-105.

Schwarze, Christoph ( $\left.{ }^{2} 1995\right)$ : Grammatik der italienischen Sprache. Tübingen, Niemeyer.

Skopeteas, Stavros et al. (2006): "Questionnaire on information structure (QUIS). Reference Manual". Interdisciplinary Studies on Information Structure. Journal des Sonderforschungsbereich 632: Informationsstruktur 4: 231-247.

Snell Hornby, Mary (1986): "Einleitung". In: Snell Hornby, Mary (Hrsg.): Übersetzungswissenschaft - eine Neuorientierung. Zur Integrierung von Theorie und Praxis. Tübingen, Francke: 9-29.

Sornicola, Rosanna (1988): "Italienisch: Pragmalinguistik/Pragmalinguistica". In: Holtus, Günter/Metzeltin, Michael/Schmitt, Christian (Hrsg.): Lexikon der Romanistischen Linguistik, Vol. 4: Italienisch, Korsisch, Sardisch. Tübingen, Niemeyer: 169-188.

Sudhoff, Stefan (2012): "Fokuspartikelinventare des Niederländischen und Deutschen". In: Rothstein, Björn (Hrsg.): Nicht-flektierende Wortarten. Berlin/Boston, de Gruyter Mouton: 203-223.

Taglicht, Josef (1984): Message and Emphasis. On Focus and Scope in English. London, Longman. 
Toury, Gideon (1995): Descriptive Translation Studies and Beyond. Amsterdam/Philadelphia, Benjamins.

Ulrych, Margherita (1997): Tradurre. Un approccio multidisciplinare. Torino, UTET.

Umbach, Carla (2001): "Restriktion der Alternativen". Linguistische Arbeitsberichte 77: 165-198.

Vermeer, Hans J. (1992): Skizzen zu einer Geschichte der Translation. Vol 2. Altenglisch, Altsächsisch, Alt- und Frühmittelhochdeutsch. Frankfurt a. M., IKO.

Wehr, Barbara (2011): "La phrase clivée en français: problèmes de description". In: Dufter, Andreas/Jacob, Daniel (Hrsg.): Syntaxe, structure informationnelle et organisation du discours dans les langues romanes. Frankfurt a. M., Lang: 189-214.

Zingarelli (2010): Lo Zingarelli on-line. Bologna, Zanichelli. [Online-Version, Letzter Zugriff: 15.08.2014]. 